\title{
ARBITRARY LAGRANGIAN-EULERIAN FINITE ELEMENT ANALYSIS OF STRAIN LOCALIZATION IN TRANSIENT PROBLEMS
}

\author{
GILLES PUAUDIER-CABOT AND LAURENT BODÉ
}

Laboratoire de Mécanique et Technologie, ENS de Cachan/CNRS/Université P et M. Curie, GRECO Géomatériaux, 61 Avenue du Président Wilson, F-94235 Cachan Cedex, France

\section{ANTONIO HUERTA}

Departamento de Matemática Aplicada III, ETS de Ingenieros de Caminos, Universitat Politècnica de Catalunya, Campus Nord C-2, E-08034, Barcelona, Spain

\begin{abstract}
SUMMARY
Non-local models guaranty that finite element computations on strain softening materials remain sound up to failure from a theoretical and computational viewpoint. The non-locality prevents strain localization with zero global dissipation of energy, and consequently finite element calculations converge upon mesh refinements to non-zero width localization zones. One of the major drawbacks of these models is that the element size needed in order to capture the localization zone must be smaller than the internal length. Hence, the total number of degrees of freedom becomes rapidly prohibitive for most engineering applications and there is an obvious need for mesh adaptivity. This paper deals with the application of the arbitrary Lagrangian-Eulerian (ALE) formulation, well known in hydrodynamics and fluid-structure interaction problems, to transient strain localization in a non-local damageable material. It is shown that the ALE formulation which is employed in large boundary motion problems can also be well suited for non-linear transient analysis of softening materials where localization bands appear. The remeshing strategy is based on the equidistribution of an indicator that quantifies the interelement jump of a selected state variable. Two well known one-dimensional examples illustrate the capabilities of this technique: the first one deals with localization due to a propagating wave in a bar, and the second one studies the wave propagation in a hollow sphere.
\end{abstract}

KEY WORDS: non-linear computational mechanics, arbitrary Lagrangian-Eulerian; mesh adaptivity; strain-softening. localization; damage mechanics; wave propagation

\section{INTRODUCTION}

Prediction of strain localization in transient dynamics is of importance for the design and verification of concrete and reinforced concrete structures subjected to impact loads or explosions, for which the response of the structure up to failure is required. For materials like concrete, failure is triggered by localization caused by strain-softening, a feature which is necessary in order to describe progressive cracking. However, before these phenomena are modelled, theoretical and computational problems should be addressed; first, a mathematically consistent model, in agreement with the mechanical behaviour of the material, must be developed for the description of the localization and the post-localization response; second, an efficient computational strategy must be used in order to capture the large local variations of the material parameters. This paper is focused on the later problems. 
From a mathematical point of view, the appearance of localization in classical rate independent continuum mechanics is associated with a change of type of the governing equations. In dynamics, the equations of motion change from hyperbolic to elliptic at the inception of localization. It has been pointed out on numerous occasions ${ }^{1,2}$ that strain-softening in classical continuum mechanics produces failure without energy dissipation (it occurs over a set of measure zero) and this is totally unrealistic. As a consequence, finite element calculations suffer from pathological mesh dependence. To overcome these difficulties, localization limiters must be implemented. Among these are the micropolar constitutive models, ${ }^{3}$ non-local models, ${ }^{2,4}$ gradient dependent models, ${ }^{5,6}$ and rate dependent models. ${ }^{7}$ These constitutive relations bear the same fundamental property: and internal length, also called intrinsic characteristic length, is introduced in the constitutive equations to limit the thickness of the shear band. Actually, this implies that two well-differentiated length scales are present in the problem: one associated to the macroscopic behaviour of the solid and one micro-scale related to the localization zone.

From a computational point of view, there is a basic underlying issue common to every constitutive model or resolution technique: the spatial interpolation of the primitive variables. The solution is desired at the micro-scale level: shear bands or, generally speaking, high localized gradients must be accurately described. This implies that the measure of the discretization (loosely speaking: the element size) must be in the order of, or even smaller than, the internal length in static and dynamic analyses. In the later, for instance, semidiscrete studies of harmonic wave propagation in a bar with a uniform mesh have shown that element sizes must be one order

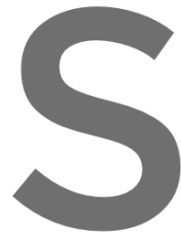
of magnitude sn other hand, the that noticed that the

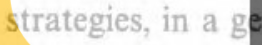

In most acade prediction of the focalization zone is needed
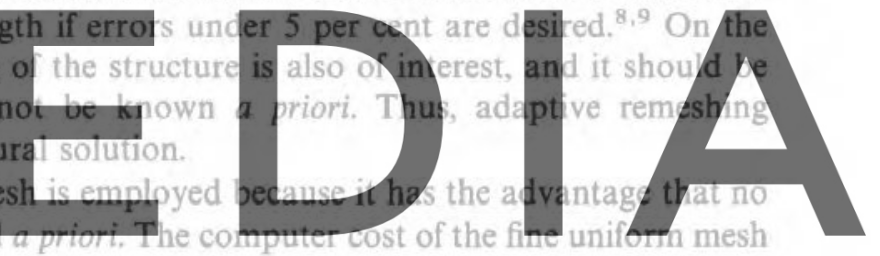

in real engineering problems is, however, totally prohibitive because the entire domain is

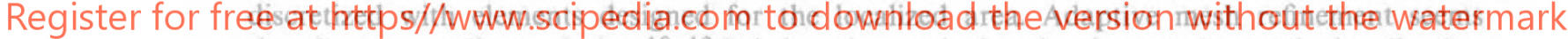
therefore an excellent solution. ${ }^{10-12}$ It is based on reducing the element size on the localization band and has proved its effectiveness in steady-state analyses. However, it needs to modify the mesh connectivity at each iteration and the elaboration of sophisticated algorithms to preclude an increase in number of elements, algorithms which are usually restricted to triangular elements. Ortiz and Quigley ${ }^{10}$ present an excellent discussion on the difficulties that have deterred an extensive application of the adaptive methods in the context of strain localization: path-dependent constitutive relations and error estimation relying, in statics, on the ellipticity of the equations which is lost at the inception of localization. Finally, methods based on spectral overlays ${ }^{13}$ have the advantage of increasing the richness of the interpolation in a particular zone without the reduction in the element size. This allows higher rates of convergence but requires an a priori knowledge of the localization area.

In this paper, another generalized spatial interpolation technique is suggested: the arbitrary Lagrangian-Eulerian (ALE) formulation. This technique which is now well established in the fluid mechanics field has recently been extended to non-linear continuum mechanics in transient analyse ${ }^{14}$ increasing the field of applicability of this formulation in the context of explicit codes. However, the ALE formulation has remained up to now restricted to the problems that naturally suggested its need: boundary motion analyses. Here, the ALE is reinterpreted as a simple numerical technique to modify the spatial interpolation introducing more degrees of freedom where they are needed. 


\section{ALE FORMULATION}

The arbitrary Lagrangian-Eulerian formulation is briefly reviewed in this section. A complete presentation may be found in. ${ }^{14-18}$ The material, spatial and referential co-ordinates denoted by $\mathbf{X}, \mathbf{x}$ and $\chi$, are respectively, related to the particles, laboratory and the kinematic reference employed. A Lagrangian formulation is employed when the reference, $\chi$, coincides with the material particles, $\mathbf{X}$; and an Eulerian formulation imposes a reference fixed in the laboratory or spatial domain. An ALE formulation allows an arbitrary motion of the reference with respect to the material and spatial co-ordinates, see Figure 1 . This is perfectly suited for large boundary motion problems in fluid ${ }^{15,19-21}$ and solid ${ }^{14,16-18,22}$ mechanics; here the same formulation is used not to account for boundary motion but to enhance (refine) the spatial interpolation in order to capture the localization areas.

The kinematics in the ALE description that link it to the classical Lagrangian and Eulerian descriptions are designed to relate material time derivatives, with referential time derivatives and spatial gradients. The fundamental equation in ALE, originally devised in Reference 21 is

$$
\left.\frac{\partial \psi}{\partial t}\right|_{\mathrm{x}}=\left.\frac{\partial \psi}{\partial t}\right|_{x}+c_{i} \frac{\partial \psi}{\partial x_{i}}
$$

where $\psi$ is any physical property, and $\mathbf{c}$ is the convective velocity which relates the material, $\mathbf{v}$, and mesh, $\hat{\mathbf{v}}$, velocities
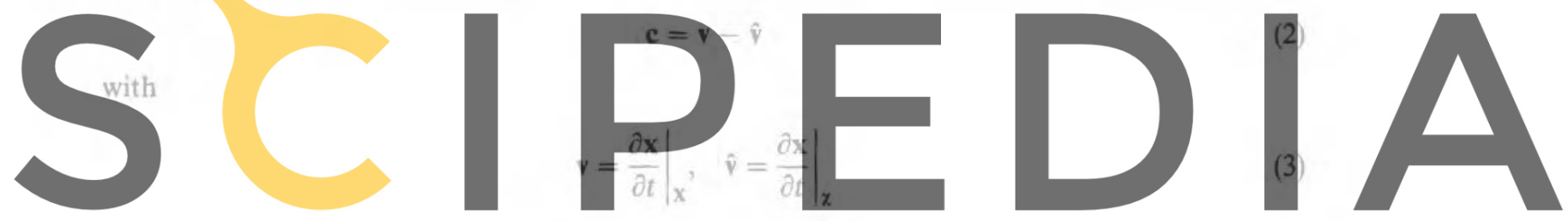

Register for free at https//www.scipedia.com to download the version without the watermark

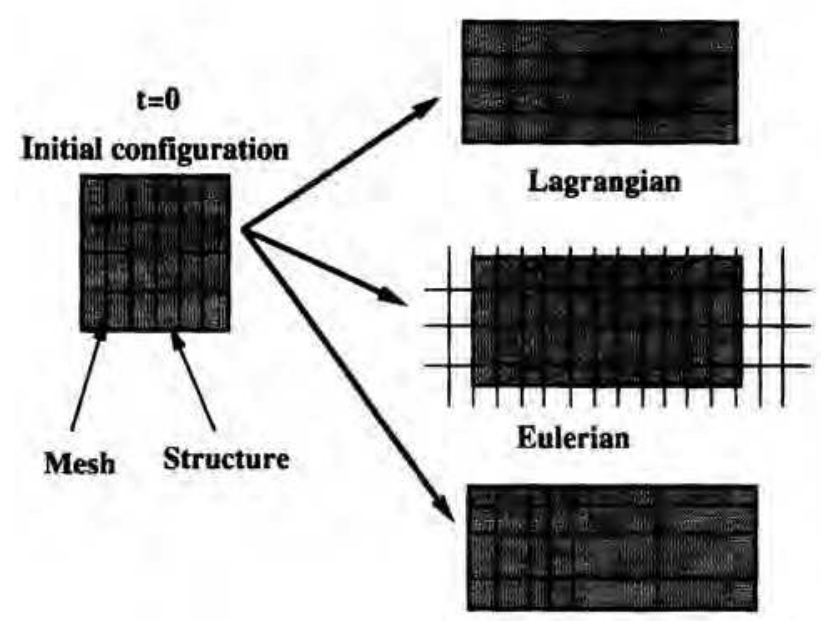

ALE

Figure 1. Schematic representations for the Lagrangian, Eulerian and ALE formulations 
Notice that $\mathbf{v}$ is the unknown particle velocity but $\hat{\mathbf{v}}$ is an arbitrarily chosen mesh velocity. The remeshing technique, described in future sections, is based on an automatic procedure to compute $\hat{\mathbf{v}}$.

With an ALE formulation, i.e. using equation (1), the equilibrium equation is rewritten in strong form as

$$
\left.\rho \frac{\partial v_{i}}{\partial t}\right|_{\chi}+\rho c_{j} \frac{\partial v_{i}}{\partial x_{j}}=\frac{\partial \sigma_{i j}}{\partial x_{j}}
$$

where $\rho$ is the density, $\sigma$ is the Cauchy stress tensor, no body forces are included, and standard indicial notation is adopted.

Apart from the conservation equations the constitutive law is also modified to account for the relative motion between mesh and particles. Any of the frequently employed rate-type constitutive equations may be written after substitution of equation (1) as

$$
\left.\frac{\partial \sigma_{i j}}{\partial t}\right|_{x}+c_{k} \frac{\partial \sigma_{i j}}{\partial x_{k}}=r_{i j}(\rho, v, \sigma)
$$

where $\mathbf{r}$ is the usual Lagrangian rate of the Cauchy stress tensor. The integration of equation (5) is readily done in a Lagrangian formulation because quadrature points at which stresses are evaluated coincide with the same material points throughout the deformation. In ALE this integration is more difficult but it is important to observe that equation (5) is a scalar equation for each stress component. Moreover, all the 'stress related variables', i.e. variables or constant fields
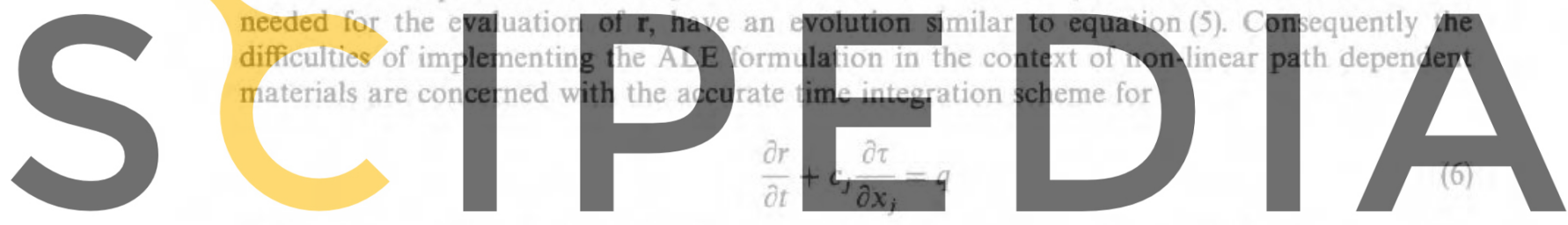

where $\tau$ is any stress or related variable and $q$ is a general non-linear source term.

freesat hittes/dway scipedial com to downlaadithe version without

Lagrangian stress is obtained by simply integrating the Lagrangian stress rate, $q$. Then, the pseudo-Lagrangian stress is convected. This step is equivalent to the interpolation or projection (between old and new meshes) needed in any adaptive mesh refinement technique. Any numerical formulation for first-order hyperbolic or conservation equations may be implemented. Here a full upwind technique is used because it can be adapted to the particular nature of the stress fields (discontinuous element to element) and to the numerical constraints (explicit code), see Reference 14 where a comparison between two formulations is presented in one- and two-dimensional examples. The detailed equations are presented in next sections.

\section{NON-LOCAL DAMAGE MODEL}

The localization limiter used in this study is the non-local damage model proposed by PijaudierCabot and Bažant. ${ }^{4}$ The stress-strain relation is identical to that of a scalar continuous damage model:

$$
\sigma_{i j}=(1-D) C_{i j k l} \varepsilon_{k l}
$$

in which $\sigma_{i j}$ and $\varepsilon_{i j}$ are the $i j$ components of the stress and strain tensors, respectively; the scalar $D$ is the damage variable which ranges from 0 initially to 1 when the material cannot sustain any stress; and $C_{i j k l}$ are the components of the elastic fourth-order tensor of the undamaged material. 
The growth of damage is defined by a loading function $f$ and its associated evolution equation. The loading function is

$$
f(\bar{Y}(\mathbf{x}), D)=\int_{0}^{\bar{Y}(\mathbf{x})} F(\mathbf{z}) \mathrm{d} \mathbf{z}-D
$$

where $F$ is a function which describes the growth of damage, and $\bar{Y}(\mathbf{x})$ is the average energy release rate due to damage at point $\mathbf{x}$. In fact, this quantity (the average energy release rate $\bar{Y}$ ) introduces the non-local nature of the model because it is defined as a weighted average of the local energy release rate, $Y$, over the entire material domain denoted as $\mathscr{V}$, namely

$$
\bar{Y}(\mathbf{x})=\int_{r} \Psi(\mathbf{s}-\mathbf{x}) Y(\mathbf{s}) \mathrm{d} \mathbf{s}
$$

with

$$
Y(\mathbf{s})=\frac{1}{2} \varepsilon_{i j}(\mathbf{s}) C_{i j k l} \varepsilon_{k l}(\mathbf{s})
$$

The weighting function $\Psi$ is a normalized bell-shaped function, i.e.

$$
\Psi(s-x)=\frac{e^{-\|s-x\|^{2} / 2 \ell^{2}}}{\int_{\mathscr{r}} e^{-\|z-x\|^{2} / 2 l^{2}} d z}
$$

where $\mathrm{t}$ is the internal length of the non-local continuum which can be estimated experimentally
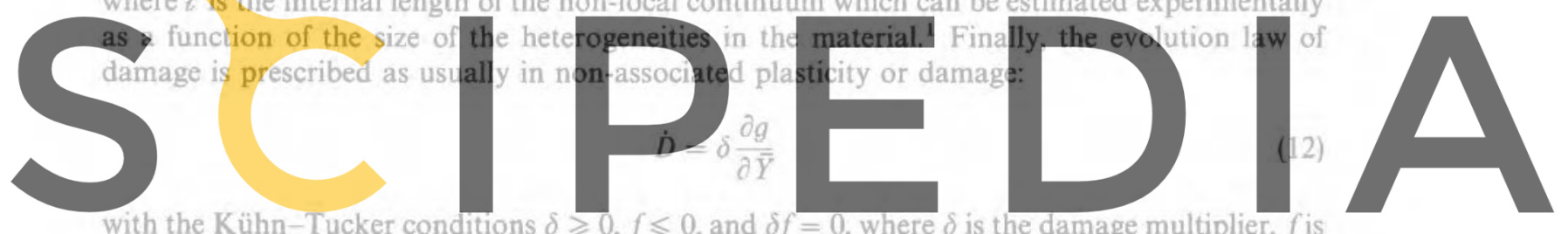

with the Kühn-Tucker conditions $\delta \geqslant 0, f \leqslant 0$, and $\delta f=0$, where $\delta$ is the damage multiplier, $f$ is defined in equation (8), and $g$ is the evolution potential which is simply the average energy release

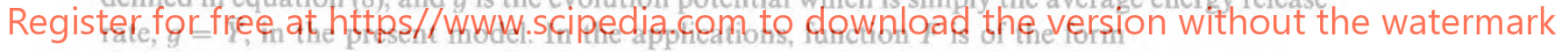

$$
F(\mathbf{x})=\frac{B}{\left[1+B\left(\bar{Y}(\mathbf{x})-Y_{0}\right)\right]^{2}}
$$

where $B$ and $Y_{0}$ are material parameters.

\section{REMESHING STRATEGY}

In the ALE description the mesh nodes move arbitrarily in the Lagrangian co-ordinate system. The mesh motion is determined once the convective velocity or the mesh velocity is chosen. In hydrodynamics and fluid-structure interaction problems the objective of the ALE formulation is to maintain regular shaped elements; consequently, the remeshing algorithms are based on geometrical considerations. In this paper, the remeshing strategy must be adequate to capture correctly the localized region, and also to introduce in it 'enough' finite elements to minimize the discretization errors whose influence in the phase velocity is demonstrated in Reference 8 .

In adaptive remeshing techniques an error indicator and a mesh optimality criterion are defined. From a general point of view, error indicators could be divided into (i) those based on the properties of the equations under study, see References 23-25 among others, and (ii) those purely related to the interpolation of the unknown variables, see References 10,11 and 26 . This extremely simple classification is independent of their ability to quantify the error or to control 
the quality of the computations. On the other hand, the optimality criterion is designed to provide the desired element size. Among the several mesh optimality criteria the most usual one is based on the equidistribution of the error indicator, but others may be also used, see for instance Reference 27.

In the context of localization problems, several error indicators have been employed. Among those based on the properties of the equations, the success of the Zienkiewicz-Zhu ${ }^{25}$ a posteriori error estimator initially developed for elastic problems induced at first its direct application to localization problems. ${ }^{12}$ However, since it is based on a standard energy norm, the loss of ellipticity of the underlying equations precludes efficient results, as Ortiz and Quigley ${ }^{10}$ demonstrated. Nevertheless, the standard energy norm or a norm based on the plastic dissipation can be used when a localization limiter is implemented, see the comparisons presented in Reference 28.

Other authors ${ }^{10,11}$ use interpolation-based estimators. While in Reference 10 the variation of the velocity field (related to the displacement interpolation error bounds in Sobolev spaces) is the error indicator, in Reference 11 the error indicator used in compressible flow computations by ${ }^{26}$ is modified for localization problems. The later error indicator is based on the quadratic departure from the piecewise linear interpolation assuming exact nodal values. Therefore, a 'key' variable for the problem, such as density in compressible flow, is chosen and the error indicator detects the ability of the continuous piecewise linear interpolation to approximate it. Second derivatives of such 'key' variable are needed. In Reference 11 instead of a single variable two functionals of field variables and their derivatives are used, one related to the plastic deviatoric

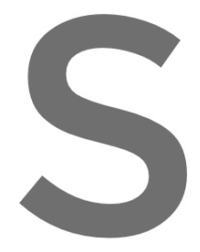
strain and the other related to the determinant of the acoustic tensor. In spite of the fact that both functionals do no have a continuous piecewise linear interpolation, that nume ric
of the second-order derivatives of internal variables such as plastic strains is que
triangular linear elements, and that without any regularization technique the finite
converges to zero width elemente along the localization zone, the results are enco
The remeshing strategy in the arbitrary Lagrangian-Fulerian formulation follows fact that both
stionable for
element mesh
a simfla line of thought: first an error indicator must be defined and then a mesh optimality criterion is chosen. With respect to the error indicator, it is important to observe that in the present problem, the which is characterized by propagating damage fronts, because the solution in this highly non-linear problem is laigely dependent on the damage localization zone. Therefore, it is assumed that the optimum mesh must concentrate elements in the neighbourhood of sharp variations of damage. Consequently, instead of using the bounded variation of the velocity field as a suitable norm of the solution, see Reference 10 , the variation of a dependent variable, damage, is taken as the relative indicator of the error estimate. Damage is a bounded field, recall that it varies between 0 initially and 1 when the material cannot sustain any stress (failure). The numerical evaluation of the mesh indicator is, in this case, extremely efficient in contrast to Reference 11 where second derivatives of the indicator involving sophisticated schemes, such as projections or variational recoveries, are needed. On the other hand, as the other error estimators based on the interpolation errors, the indicator only provides a control of the quality of the mesh. That is, it simply detects when the mesh is too coarse and a minimum admissible element size must be given and related to the maximum value of the indicator to determine the desired element sizes in the rest of the domain. Nevertheless, the ALE formulation is perfectly suited for such relative error estimates, because it does not introduce new elements, in fact the motion of the computational frame may be also based on a relative measure of the element size.

In one-dimensional problems, damage is known at the Gauss points but the mesh indicator, denoted $\mathscr{K}$, is defined at each nodal point as a function of the absolute value of the jump of damage across elements; in higher dimensions, generalizations similar to those presented in 
Reference 10 could be used. That is,

$$
\mathscr{K}\left(x_{i}\right)=a\left[D\left(x_{i}\right)\right]+b, \quad\left[D\left(x_{i}\right)\right]=\left|D^{i+1}-D^{i}\right|
$$

where $a$ and $b$ are two parameters, $x_{i}$ is the co-ordinate of node $i$ and $D^{i}$ denotes the damage at element $i$.

In order to equidistribute the error indicator $\mathscr{K}$ over the entire domain the following equation must be solved:

$$
\frac{\partial}{\partial \chi}\left[\mathscr{K}(x) \frac{\partial x}{\partial \chi}\right]=0
$$

It is based on the classical elliptic mapping of the co-ordinate lines, see Reference 29 and it is automatically generalizable to higher dimensions. In equation (15) the indicator $\mathscr{K}$ may be regarded as a diffusion coefficient known at each nodal point. In fact, equations (14) and (15) with the known position of the domain boundary define a Dirichlet problem which has a simple physical interpretation: the error indicator $\mathscr{K}$ times the element measure must be kept constant over the domain. The parameters $a$ and $b$ in equation (14) control the relative element size. It can be easily shown that the ratio of the maximum over the minimum length of the elements throughout the mesh is bounded by $1+a / b$ (see Appendix).

Equation (15) induces a non-linear system which must be solved iteratively at each time step, once the error indicator is computed. This is certainly a major drawback because fast transient
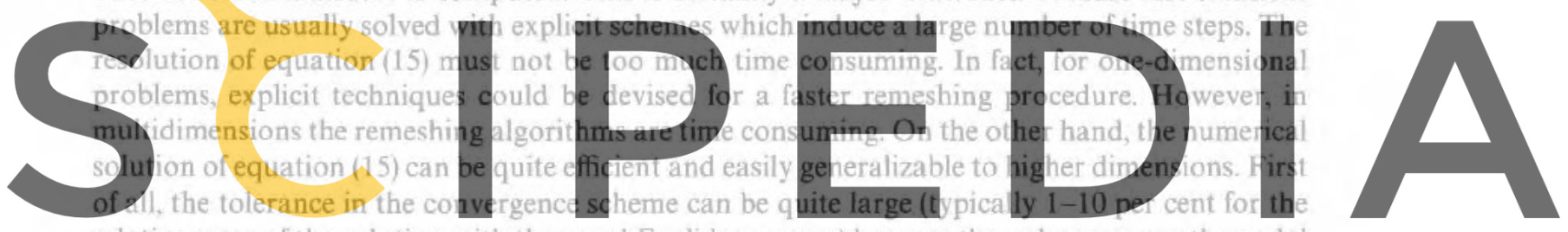

relative error of the solution with the usual Euclidean norm) because the unknowns are the nodal Register for free at https/ www.scipedia.com to downlo momethe version without the watermark notice that this is an evolutionary problem, thus the initial approximation of the solution in the non-inear system of equations is relativeiy ciose to the inai solution because the time steps are small. And third, efficient techniques such as inverse Broyden method with line search can be implemented. In all the applications, the number of iterations is relatively small: usually two and always less than five. Nevertheless, the development of an adequate explicit remeshing algorithm which will serve the same purpose is the subject of further research.

When the error indicator is only a function of damage, remeshing starts after damage has started to grow in one element. If damage and localization occur at time steps close to each other, the inception of the localization will be controlled by the initial mesh. Indeed, since damage has not grown sufficiently, the mesh may be still too coarse. This initial deficiency of the discretization may perturb significantly the subsequent distributions of damage. In order to avoid this problem, remeshing can also be performed starting at the beginning of the computation. For this, the mesh indicator is taken as a function of the second invariant of strain. In a one-dimensional formulation it simply reduces to

$$
\mathscr{K}\left(x_{i}\right)=a \llbracket \hat{\varepsilon}\left(x_{i}\right) \rrbracket+b, \quad \llbracket \varepsilon\left(x_{i}\right) \rrbracket=\left|\hat{\varepsilon}^{i+1}-\hat{\varepsilon}^{i}\right|
$$

where $\hat{\varepsilon}^{\prime}$ is the normalized second invariant of the strain at element $i$. This error indicator is used up to the inception of damage when the previously defined one, equation (14), is activated. In the applications, both cases are implemented. 


\section{FINITE ELEMENT IMPLEMENTATION-EXAMPLES}

In order to illustrate the capabilities of this computational method, two well-known problems which deal with localization due to wave propagation are considered. The first one is a rod subjected to constant velocity boundary conditions. ${ }^{1}$ The second example deals with the propagation of a pressure wave in a spherically symmetric structure. This example was proposed by Belytschko et al., ${ }^{2}$ and further studied in Reference 6 . These two problems are one-dimensional computations which are aimed at exhibiting the main properties of the continuum description and the computational strategy.

The one-dimensional weak form of the momentum equation is

$$
\left.\int_{0}^{L} \rho \delta u \frac{\partial v}{\partial t}\right|_{x} \mathrm{~d} x+\int_{0}^{L} \rho \delta u c \frac{\partial v}{\partial x} \mathrm{~d} x+\int_{0}^{L} \frac{\partial \delta u}{\partial x} \sigma \mathrm{d} x=0
$$

where the first integral is classical, it is associated to the local increment of velocity; the second one is related to the convective effects due to the relative motion between mesh and particles; and the last one is the usual internal force term. Note that according to this equation, accelerations are the time derivatives of the particle velocity, $v$, holding the reference frame fixed (not the particle, nor the laboratory). However, the spatial gradient of the velocities corresponds exactly to the classical Lagrangian strain (under the small strain assumption).

An explicit central difference scheme has been used for the time integration of the momentum
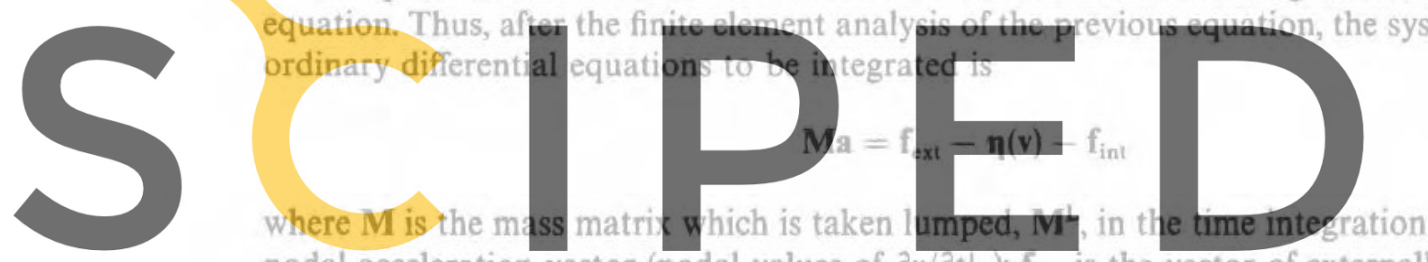

nodal acceleration vector (nodal values of $\partial v /\left.\partial t\right|_{\chi}$ ); $f_{\text {ext }}$ is the vector of externally applied loads; $\eta(v)$ is the convective vector, it is related to the second term of equation (17); and $\mathbf{f}_{\text {int }}$ is the usual Register for freeratihttps/dWWWwssipedia.Gem tQedpwnload the version without the watermark

The convective term depends on the arbitrarily imposed mesh velocity and also on the unknown particle velocity: it is non-linear. This non-linearity which is easily overcome implementing an explicit algorithm precludes second-order accuracy of the time integration scheme for constant time steps. However, the relative influence of the convective term in equation (18) for all the studied cases indicates that almost second-order accuracy is achieved.

The time integration algorithm is detailed in Box 1. It shows that very simple modifications are needed in a classical explicit code to introduce the ALE formulation. Apart from the implementation of the convective term already discussed, it is necessary to evaluate the new nodal coordinates and to update the strain and damage.

In order to compute the convective velocities, the new nodal co-ordinates are evaluated according to the remeshing strategy. Here, this is done at each time step but remeshing can be activated whenever the user desires. The finite element integration of equation (15), a non-linear Laplace equation with Dirichlet boundary conditions (the known positions of the contour nodes), induces the following non-linear system of equations:

$$
\mathbf{L}(\mathbf{x}) \mathbf{x}=\mathbf{b}
$$

where the unknowns are the new nodal positions. As cited previously, an inverse Broyden algorithm with one iteration per line search has been implemented. 
Box 1: Flow chart of the ALE finite element program

\section{Algorithm for the ALE explicit code}

1. Initialization and initial conditions

2. Loop over time steps

At time $t^{n}$ :

(a) Given $\varepsilon^{n}$, compute $\sigma^{n}$ and $D^{n}$ use equations (7)-(13).

(b) Given the spatial co-ordinates $\mathbf{x}^{n}$, compute the internal forces and the mass matrix at $t^{n}$.

(c) From the particle, $\mathrm{v}^{n-1 / 2}$, and convective velocities, $\mathrm{c}^{n-1 / 2}$, compute the convective force, $\boldsymbol{\eta}^{n-1 / 2}(\mathbf{v})$, at $t^{n-1 / 2}$.

(d) Solve the equation of motion with the lumped mass matrix and compute the accelerations at $t^{n}$.

$$
\mathbf{M}^{L} \mathbf{a}^{n}=\mathbf{f}_{\mathrm{ext}}^{n}-\eta^{n-1 / 2}(\mathrm{v})-\mathbf{f}_{\text {int }}^{n}
$$

(e) Compute new velocities and strains

- Loop over nodes

$$
\mathrm{v}^{n+1 / 2}=\mathrm{v}^{n-1 / 2}+\Delta t \mathrm{a}^{n}+\mathcal{O}\left(\Delta t^{2}\right)
$$
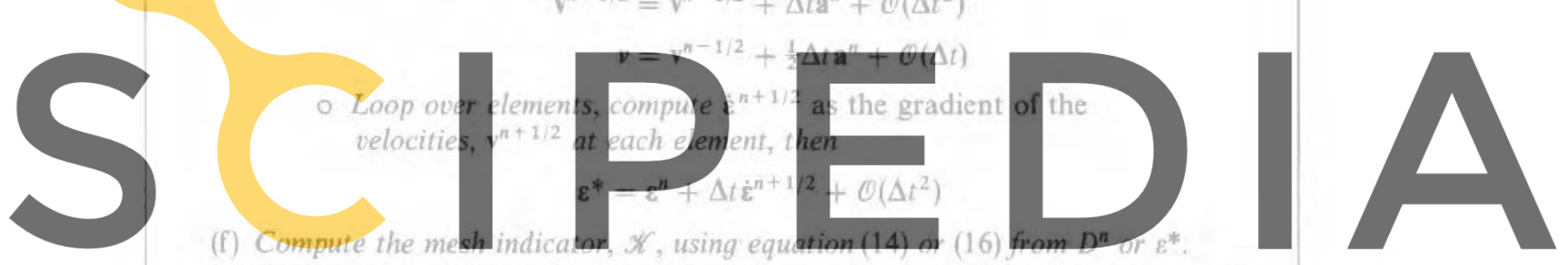
Register for free athttps/\%wivw:scipedia.com to download the version without the watermark

$$
\begin{aligned}
c^{n+1 / 2} & =v^{n+1 / 2}-\left(\frac{\mathbf{x}^{n+1}-\mathrm{x}^{n}}{\Delta t}\right)+\Theta\left(\Delta t^{2}\right) \\
\mathbf{c}^{n} & =\mathbf{v}^{n}-\left(\frac{\mathbf{x}^{n+1}-\mathbf{x}^{n}}{\Delta t}\right)+\mathcal{O}(\Delta t)
\end{aligned}
$$

(h) Update strain and damage at the new nodal positions using equation (23).

3. Compute the next time step, go to 2 .

Once the new mesh co-ordinates are obtained, the convective nodal velocities are computed:

$$
\mathbf{c}\left(t+\frac{1}{2} \Delta t\right)=\mathbf{v}\left(t+\frac{1}{2} \Delta t\right)-\left[\frac{\mathbf{x}(t+\Delta t)-\mathbf{x}(t)}{\Delta t}\right]
$$

Then, the state variables, strain and damage, can be transported to the new mesh co-ordinates. In fact, the integration of equation (6) is done by a split step technique. The Lagrangian part is computed with a usual central difference scheme for strains

$$
\varepsilon\left(t^{*}\right)=\varepsilon(t)+\Delta t \dot{\varepsilon}\left(t+\frac{1}{2} \Delta t\right)
$$


where $\dot{\varepsilon}$ is simply the vector of spatial gradients of the particles velocities, $\mathbf{v}$, at the Gauss points; and $t^{*}$ is related to a middle instant between $t$ and the end of step $t+\Delta t$. In order to obtain the final values, the convection due to the mesh motion must be evaluated, see equation (6). On the other hand, for the damage variable the Lagrangian update is directly evaluated knowing the strain field at the desired instant and the damage field at the previous step. ${ }^{4}$

Then, the convection part which is related to the right-hand side of equation (6) is computed in both cases using a full upwind first-order time integrator. A second-order Lax-Wendroff technique can also be implemented, but the results are almost identical ${ }^{14}$ Both take into account the discontinuous nature of the state variables and require, for stability, the enforcement of the local Courant condition

$$
\frac{c_{e} \Delta t}{h_{e}} \leqslant 10
$$

where $c_{e}$ and $h_{e}$ are the average convection velocity and the length at element $e$. Note that the classical numerical difficulties associated to the Riemann problem defined by equation (6) such as unstable oscillations and dispersion are drastically reduced because the convection velocity in (6) is associated to the mesh motion and it is usually much smaller than the stress wave speeds which govern the time step in explicit codes. Therefore, the time step limitation imposed by equation (22) is not restrictive. The full upwind technique, which is generalizable to higher dimensions and takes into account the incoming and outgoing information along the characteristic lines, has a simple expression in the one-dimensional case. Denote by $\tau_{e}(t)$ the state variable at the Gauss point of element $e$ of length $h_{e}$ whose godes are identifil by the local indices 1 and 2 . The full
upwind integration is simply
$\tau_{e}(t+\Delta t)=t_{e}\left(t^{*}\right)-\frac{\Delta t}{h_{e}}\left\{\frac{1+g^{\prime} g n\left(c_{1}\right)}{2}\left[\tau_{t}-\tau_{e-1}\right]+c_{2} \frac{1-\operatorname{sign}\left(c_{2}\right)}{2}\left[\tau_{e+1}-\tau_{e}\right]\right\}$ where the adjacent elements are referred by $e-1$ and $e+1$. Note that it is not mandatory to do

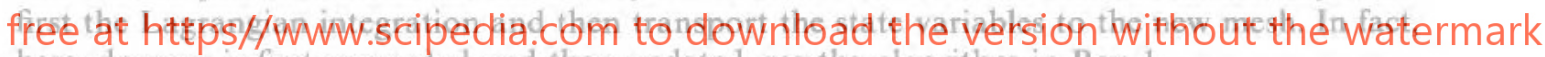
here, damage is first convected and then updated, see the algorithm in Box 1 .

\section{Bar problem}

The implementation of the non-local damage model in a one-dimensional finite element code is presented in Reference 4 where the objectivity of the discretization for this constitutive model was demonstrated. Figure 2 shows the response of the material under uniaxial tension. The material constants are taken as follows: the elastic modulus $E$ is $32000 \mathrm{MPa}$, the poisson ratio $v$ is $0 \cdot 2$, the density $\rho$ is $2.5 \times 10^{3} \mathrm{~kg} / \mathrm{m}^{3}$, and the material parameters $Y_{0}$ and $B$ are taken as $1.50 \times 10^{-4} \mathrm{MPa}$ and $9.27 \times 10^{3} \mathrm{MPa}^{-1}$, respectively. This set of parameters corresponds to concrete with an average strength. The bar has a cross section of $1 \mathrm{~mm}^{2}$ and a characteristic length $\ell=1 \mathrm{~mm}$. The velocity at the extremities is such that a constant strain wave of amplitude $0.75 \varepsilon^{*}$ (where $\varepsilon^{*}$ is the strain measured at the peak stress) is propagated. These waves meet at $t^{*}$ in the middle of the bar where localization occurs. Figure 3 shows a schematic problem statement.

Two bars of different lengths, 40 and $160 \mathrm{~mm}$, are discretized in nine elements of constant and uniform lengths and their results are compared with a 'reference solution'. This reference is obtained with a sufficient number of elements (199 elements) of constant length and fixed throughout the calculation. These two cases are studied because the ratio between the localization width and the initial mesh size are very small, in the order of 1.6 and 0.4 , respectively, while 


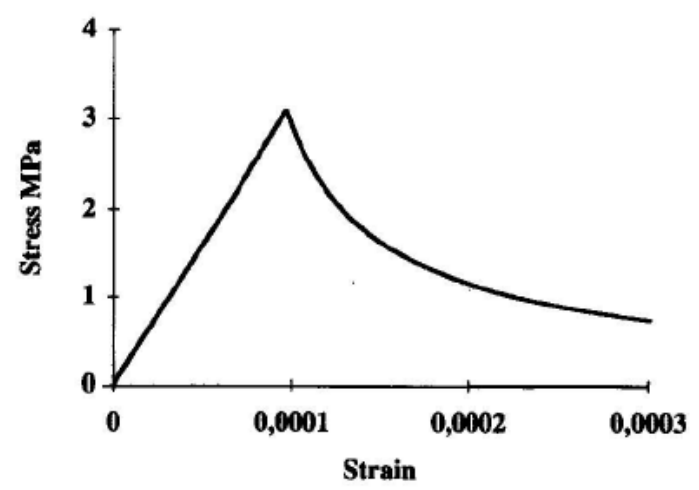

Figure 2. Concrete response under uniaxial tension

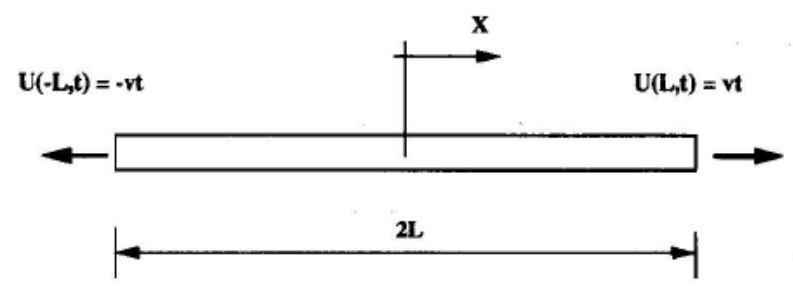

Figure 3. Wave propagation in a bar: problem statement

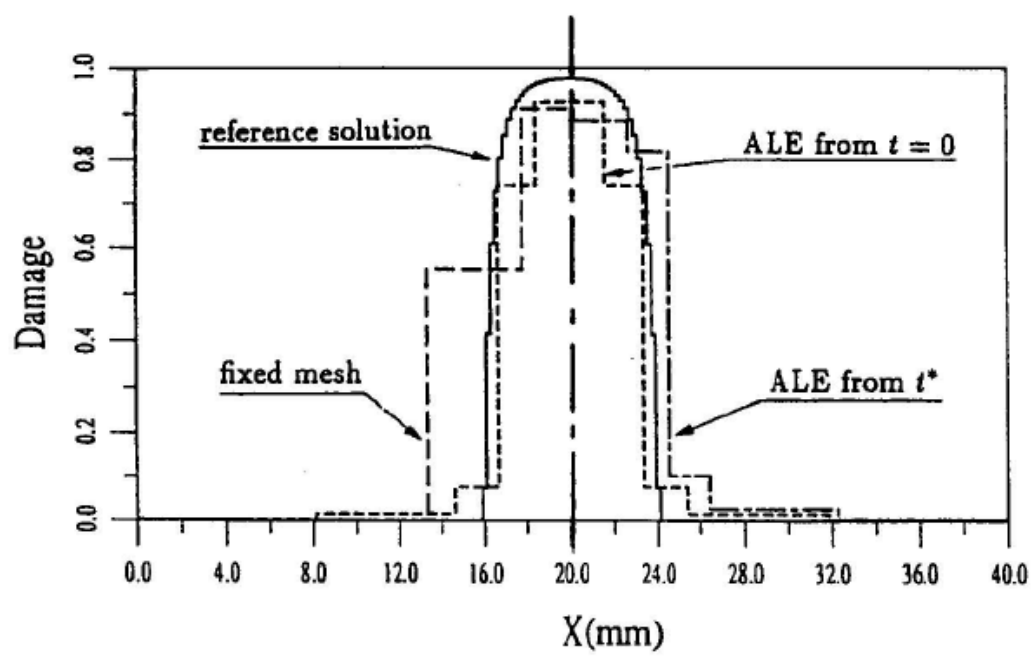

Figure 4. Wave propagation in a bar: damage profiles at $t=1.5 t^{*}$

the reference case has ratios of 33 and 9 . Notice that in the second case, the localization width determined by the reference solution is less than half the size of the initial descretization.

Figure 4 compares the damage profiles obtained at $t=1.5 t^{*}$ with the reference solution, the fixed mesh, an ALE computation with remeshing starting at $t^{*}$, and an ALE computation with remeshing from $t=0$. The length of the bar is $40 \mathrm{~mm}$. When remeshing starts at $t^{*}$, the mesh 
indicator is a function of the jump of damage, equation (14). However, when remeshing starts at $t=0$, the mesh indicator depends on the strain jump, equation (16), until damage starts to grow, then the error indicator is defined by equations (14). The remeshing parameters in equation (14) and (16) are $a=13$ and $b=1$. Compared with the reference solution, the fixed mesh clearly overestimates the localization width. On the other hand, both ALE solutions are close to the reference one.

It can be noticed that the size of the element where localization starts has some influence in the results. In fact, this difference between both ALE solutions decreases and becomes unnoticeable as the number of elements is augmented. The influence of remeshing starting before or after localization is better seen in Figure 5, where the same cases are shown at $t=1 \cdot 2 t^{*}$. In this figure both the ALE from $t^{*}$ and the fixed mesh clearly overpredict the localization width. The nodal points remain fixed if damage is constant; upon a sudden variation of the damage field, that is at the inception of localization, the finite element mesh is too coarse for a good approximation. The motion of the ALE mesh corrects this error in the following time-steps but the history-dependent constitutive equation (the rate of damage is always positive or zero) and the explicit solver for the equilibrium equation precludes a better approximation shortly after localization appears. Figure 6 presents at $t=1.5 t^{*}$ and for the same cases the element length along the bar corresponding to the damage profiles on Figure 4. As expected, the element size is reduced (half of the initial mesh) near the damage front, and it is augmented accordingly away from the localized area. Finally, Figure 7 shows the damage profiles for the $160 \mathrm{~mm}$ bar. In this extreme case where the localization width is smaller than the initial element length the influence of the remeshing is clear. The ALE computation with remeshing starting at $t=0$ captures adequately the localized area.

\section{Spherically symmetric problem}

The second test case deals with the propagation of a pressure wave in a hollow sphere. The internal radius of the sphere is $R_{2}=10 \mathrm{~mm}$ and the external one is $R_{1}=100 \mathrm{~mm}$. Figure 8 shows the problem statement. A uniform pressure is applied on the exterior surface of the hollow sphere. Due to the spherical symmetry, the stresses increase as the stress wave propagates towards the centre of the sphere. When the peak strength is reached, localization occurs over an interior

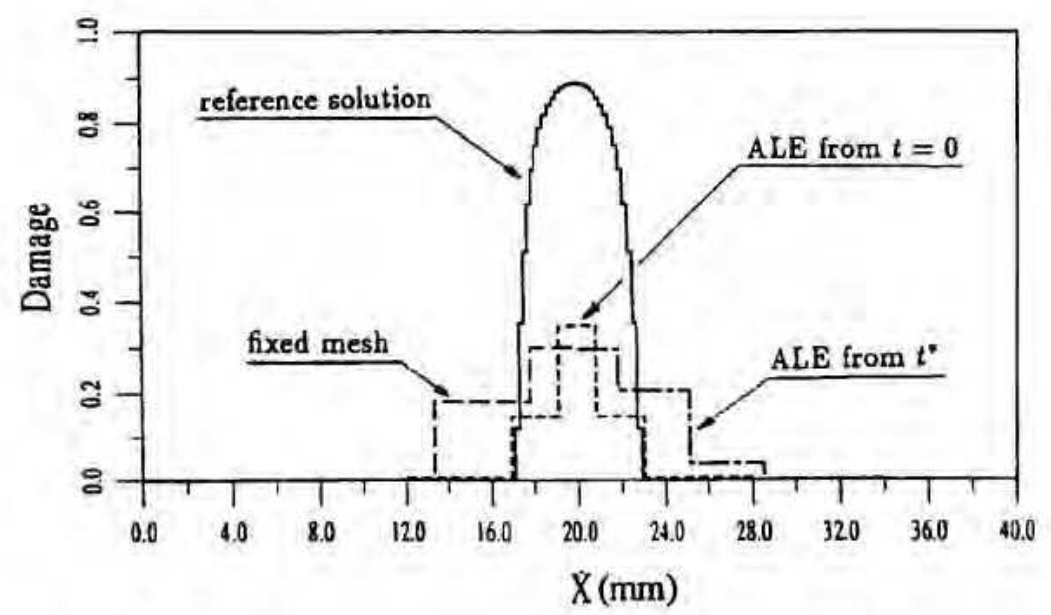

Figure 5. Wave propagation in a bar: damage profiles at $t=1.2 t^{*}$ 


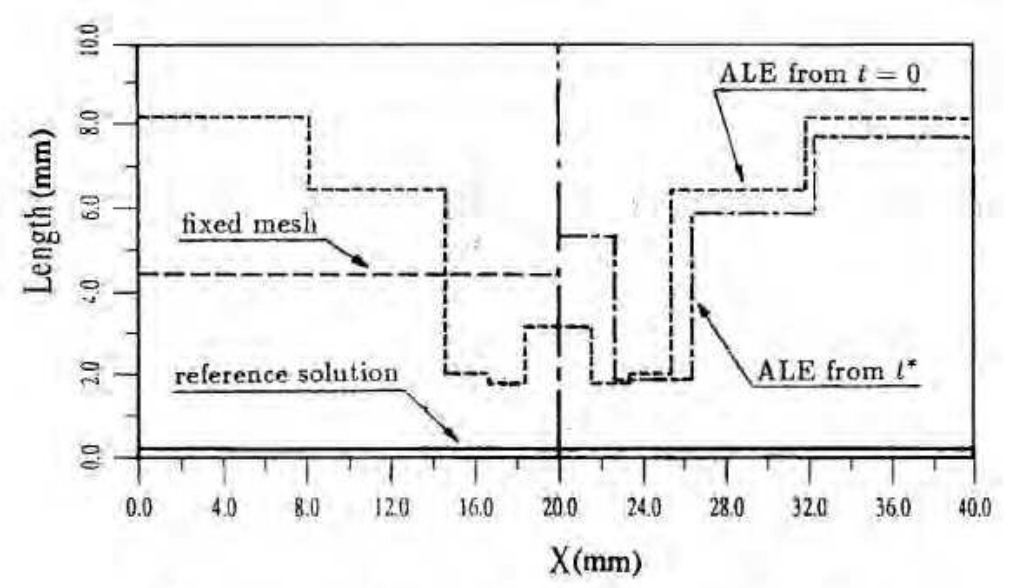

Figure 6. Wave propagation in a bar: element lengths at $t=1 \cdot 5 t^{*}$

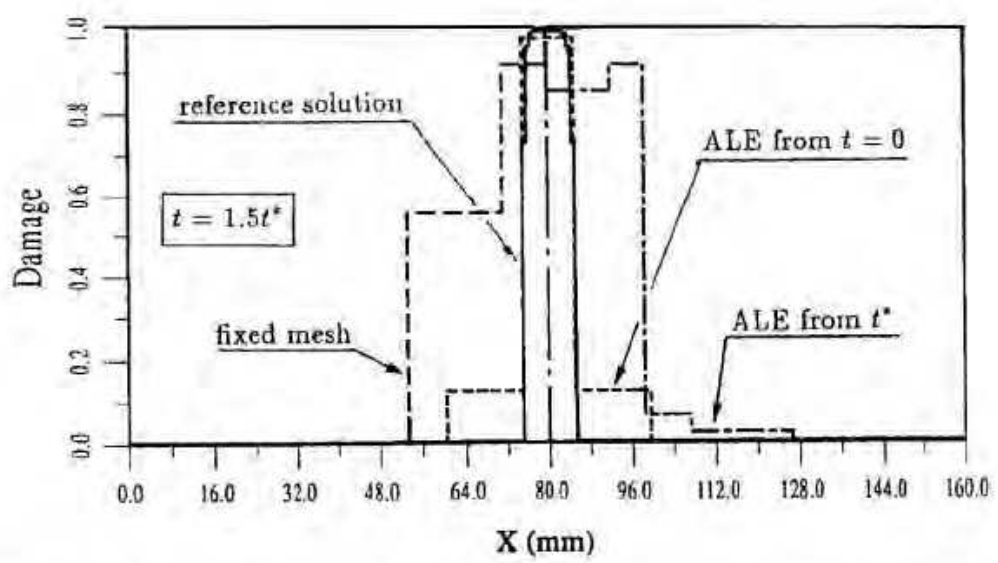

Figure 7. Wave propagation in a bar: damage profiles at $t=1.5 t^{*}$ for the $160 \mathrm{~mm}$ bar

surface of the sphere. The material constants used for this problem are: $E=1 \mathrm{MPa}$, $G=1 \times 10^{6} \mathrm{MPa}, \rho=1 \mathrm{~kg} / \mathrm{mm}^{3}$. They are identical to the constants chosen in Reference 6 . The governing equations for this problem are standard and can be found in the same reference. The constants for the evolution law of damage are $B=2 \mathrm{MPa}^{-1}, Y_{0}=0.5 \mathrm{MPa}, \ell=2.5 \mathrm{~mm}$, and the applied external pressure is $p=0.6 \mathrm{MPa}$.

The implementation of the non-local damage model in the finite element code requires the calculation of the average of the damage energy release rate $\bar{Y}$ at every Gauss point using equation (9). This equation is, in fact, an integration over the entire three-dimensional domain. However, it can be simplified to a one-dimensional computation if $R$ (distance to the centre of symmetry of any Gauss point where $\bar{Y}$ is evaluated) is sufficiently large compared to the internal length $\ell$ of the continuum, then it is well approximated by

$$
\bar{Y}(R)=\int_{R_{2}}^{R_{1}} \frac{\mathrm{e}^{(R-r)^{2} / 2 \ell^{2}}}{\int_{R_{2}}^{R_{1}} \mathrm{e}^{(R-\xi)^{2} / 2 \ell^{2}} \mathrm{~d} \xi} Y(r) \mathrm{d} r
$$




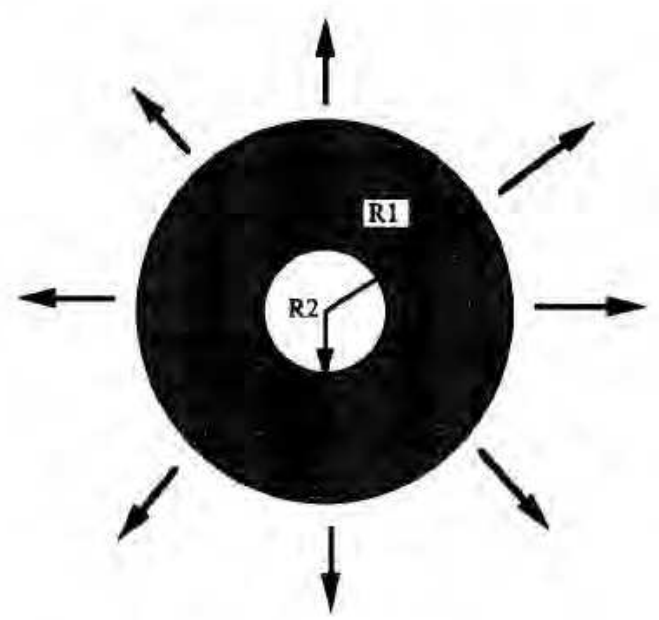

Figure 8. Wave propagation in a hollow sphere: problem statement

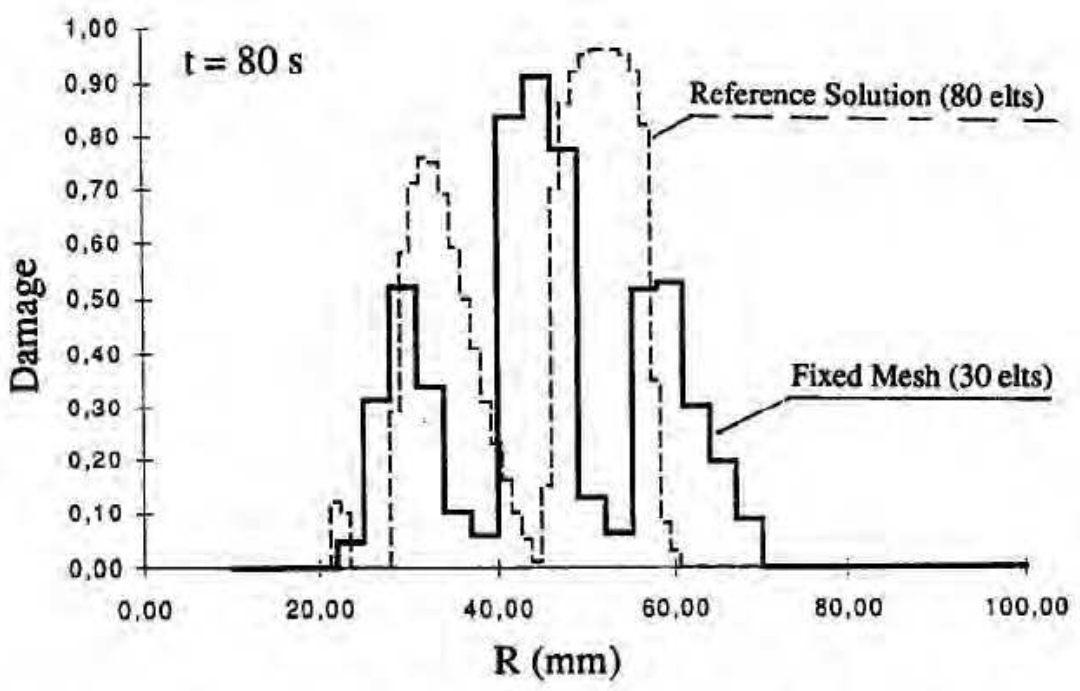

Figure 9. Wave propagation in a hollow sphere: comparison of the damage profiles between the reference solution and the fixed mesh computation at $t=80 \mathrm{~s}$

This approximation has been used in the finite element calculations. Following the previous discussion, remeshing starts at $t=0$ and it is related to the volumetric strain, equation (16), up to the inception of damage when remeshing is then controlled by damage itself, equation (14).

The reference solution has been obtained with a uniform mesh of 80 elements and a time step $\Delta t=0.5 \mathrm{~s}$. Localization appears at $t^{*}=52 \mathrm{~s}$. Figure 9 compares the distribution of damage at $t=80 \mathrm{~s}$ obtained with the reference solution and with a fixed uniform mesh of 30 elements. Notice in the reference solution that two localized areas are clearly developed and a third one is appearing. Several localized areas were expected: as the stress wave propagates to the centre it is amplified, therefore when it reaches the peak stress localization is triggered; nevertheless, loading 
waves in this dispersive medium can still propagate after localization has started, consequently the stresses are again amplified and trigger the formation of additional localized surfaces. Therefore several localization zones may appear before the wave is reflected on the interior surface at $R=R_{2}$. Note that the exact position of the localization zones is not known a priori by symmetry considerations as in the bar problem. It is important to observe that the damage distribution obtained with the coarser mesh is quite different from the reference solution. Three localization areas are clearly developed and none coincides with the positions of the reference ones. This proves the inherent difficulties of this particular problem with respect to mesh distribution.

The ALE analysis uses the same initial uniform mesh with 30 elements. Figure 10 shows a comparison between the ALE results and the reference solution at $t=60,70$ and $80 \mathrm{~s}$. Although better agreement between both calculations is observed, several qualitative differences still persist, the most important one being a slight translation of the distribution of damage towards the centre of the sphere. In view of Reference 6 it is important to remark that in the present explicit time integration no artificial damping is introduced in the fixed mesh computations; therefore, in the reference solution, spurious oscillations appear from one element to the next. On the other hand, in the ALE analysis the full upwind scheme employed for the integration of the convection term, equation (23), introduces some numerical damping. Therefore in the fixed mesh studies, such as the one with the refined mesh of 80 elements, the oscillations in the undamaged material associated to high frequencies tend to trigger localization sooner, that is, farther from the centre of the sphere. In order to suppress these oscillations, a stiffness proportional damping, similar to Reference 6, is introduced only in the non-ALE computations. The last term in equation (17) associated to the internal force is modified and the equilibrium equation is rewritten as

$$
\left.\int_{0}^{L} \rho \delta u \frac{\partial v}{\partial t}\right|_{x} \mathrm{~d} x+\int_{0}^{L} \rho \delta u c \frac{\partial v}{\partial x} \mathrm{~d} x+\int_{0}^{L} \frac{\partial \delta u}{\partial x} \sigma \mathrm{d} x+\beta \int_{0}^{L} \frac{\partial \delta u}{\partial x} \dot{\sigma} \mathrm{d} x=0
$$

where $\beta$ controls the amount of damping.

The reference solution is re-evaluated with added damping $(\beta=0 \cdot 1)$, as well as the analysis with the fixed 30 element mesh, the differences between both computations are similar to those shown in Figure 9. On the other hand, in Figure 11 it is shown that very good agreement is obtained between the ALE results $(\beta=0)$ and the reference solution with added damping $(\beta=0 \cdot 1)$. This shows the effectiveness of the ALE formulation. It is important to observe that this example is particularly demanding for the ALE formulation. Note that damage is detected, for every localized area, in coarse elements compared to the reference solution This explains the lower maximum values of damage during the generation of the bands (in a similar manner as in the bar problem). For instance the second band in Figure 11 (b) just appeared at $t=66 \mathrm{~s}$ (eight time-steps before the representation). Moreover, the remeshing tends to concentrate elements around the first localized area until the second band is detected. Consequently the mesh motion must be reversed to concentrate elements also on the second band which is correctly captured, see Figure 11(c). Finally, Figure 11 also shows that the ALE damage distributions have a small bias towards the centre of the sphere compared to the reference solution, this is due to the numerical smoothing of the sharp damage fronts introduced by the updating algorithm.

Figure 12 shows the evolution of the mesh with time. In these plots each element is represented by a rectangle whose side is proportional to the actual length. At $t=40 \mathrm{~s}$ the mesh indicator is still computed according to the volumetric strain, the ratio between the lengths of the smallest element to the largest one is approximately 3 . At $t=80 \mathrm{~s}$ damage is well developed and this ratio 
G. PIJAUDIER-CABOT, L. BODÉ AND A. HUERTA
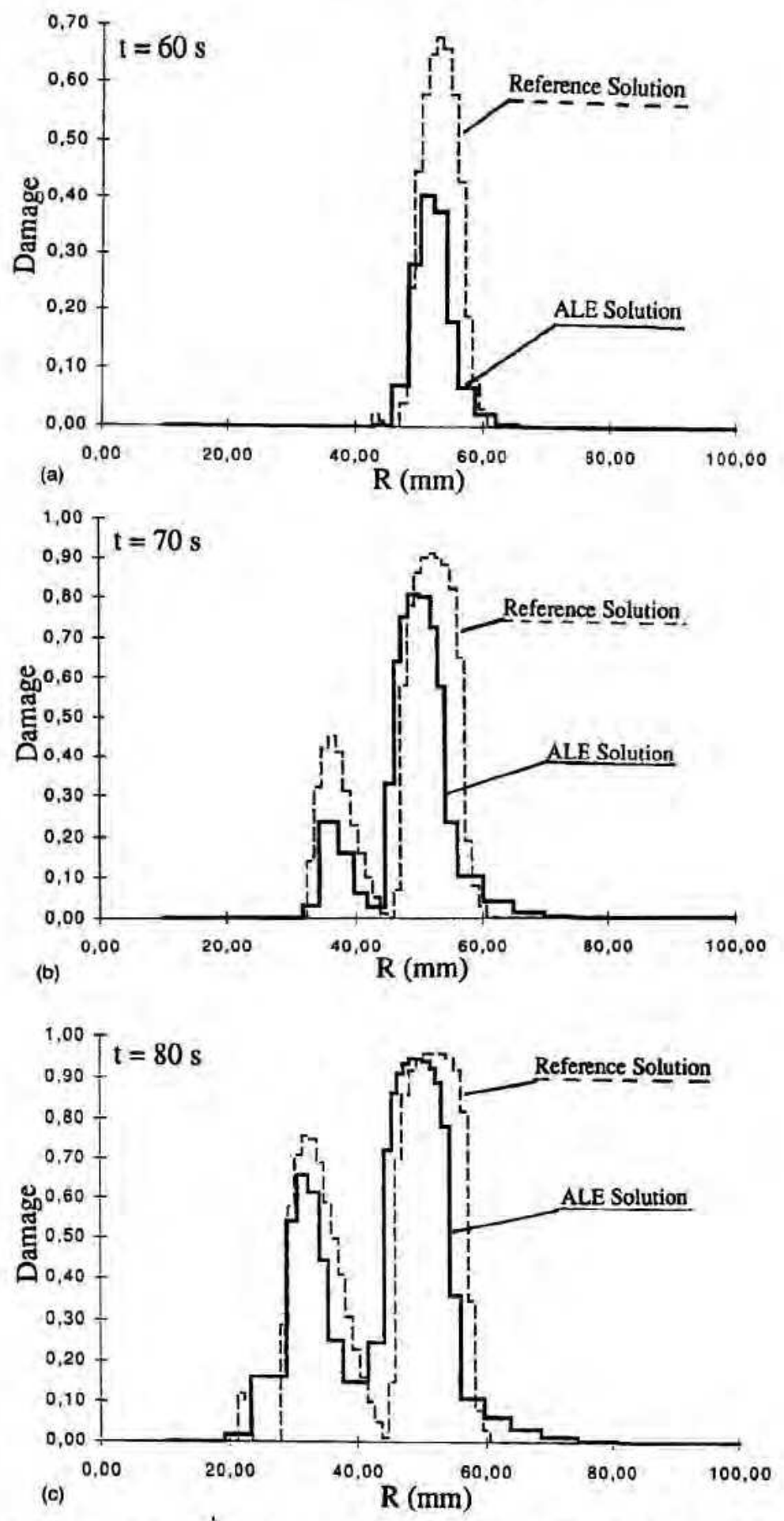

Figure 10. Wave propagation in a hollow sphere: comparison of the damage profiles between the reference solution and the ALE computation at (a) $t=60 \mathrm{~s}$, (b) $t=70 \mathrm{~s}$, and (c) $t=80 \mathrm{~s}$ 

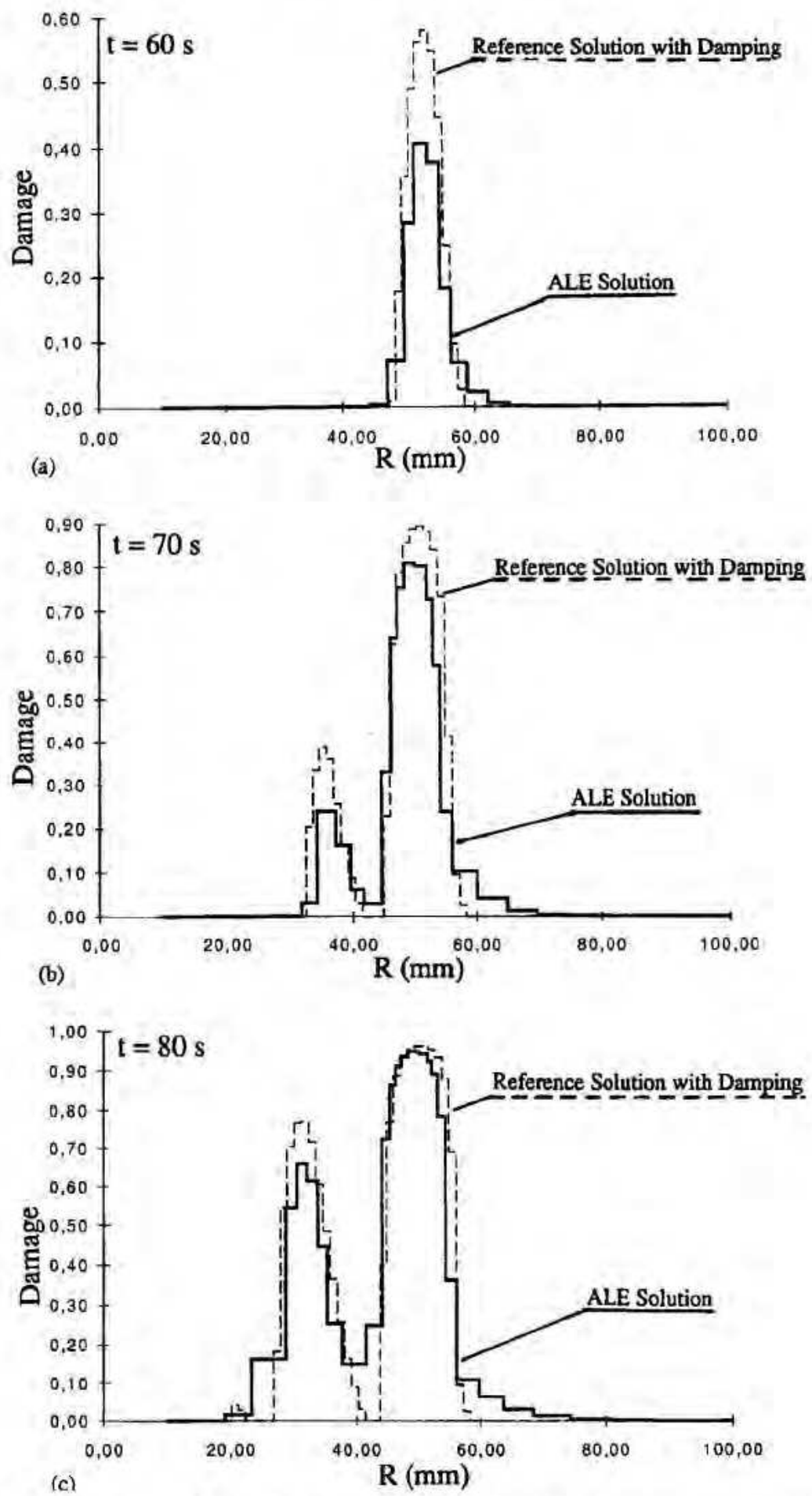

Figure 11. Wave propagation in a hollow sphere: comparison of the damage profiles between the reference solution with damping and the ALE computation at (a) $t=60 \mathrm{~s}$, (b) $t=70 \mathrm{~s}$, and (c) $t=80 \mathrm{~s}$ 


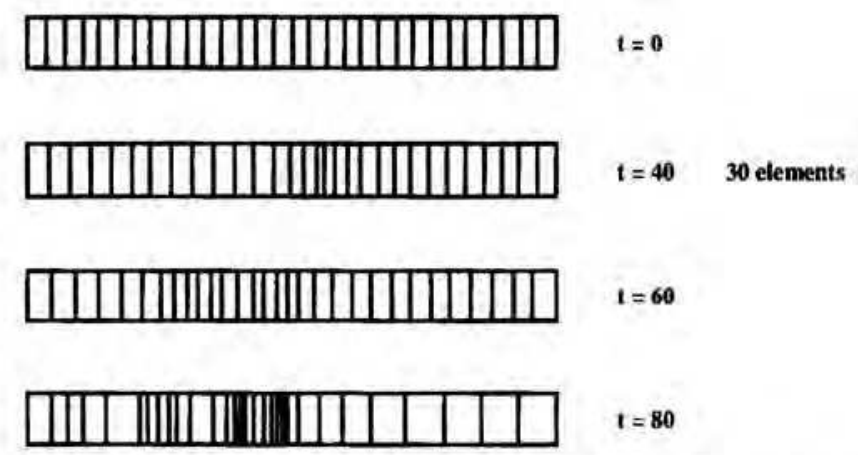

Figure 12. Wave propagation in a hollow sphere: evolution of the mesh in the ALE solution

is almost 11. The agreement between ALE and reference solutions shows that the strain and damage distributions are not distorted by the variations in the mesh indicator or in the size of the elements.

\section{CONCLUSIONS}

The arbitrary Lagrangian-Eulerian formulation is one of the techniques that can be applied to strain localization analysis in fast-transient problems. In the ALE formulation, the mesh nodes move independently of the material particles, hence the element size can be conveniently adjusted in order to capture strain localization. This formulation has several interesting properties: the mesh connectivity and the number of degrees of freedom remain constant during the computation. Furthermore, the remeshing itself is imbedded in the equation of motion which is not the case in other adaptive remeshing technique to better capture the localization area. Although only one-dimensional examples are presented, the formulation is developed to allow a further generalization to problems in two and three dimensions.

A simple remeshing strategy based on the equidistribution of the jump of material state variables (strain or damage) has been chosen in order to obtain an accurate solution. The remeshing equation reduces to a non-linear system of equations. The resolution of these equations, in spite of the effectiveness of the algorithm and the relaxed convergence requirements, is time consuming and requires further study in the context of fast-transient codes. On the other hand the strain and damage update, necessary to account for the mesh motion, is very simple and efficient.

Two test cases have been presented where good approximations are achieved with meshes whose element length is initially much larger than the internal length of the material (which scales the localization zone). When remeshing starts at the beginning of the calculation, the element size is automatically adjusted in order to obtain strain localization at the correct location. Accurate capturing of the propagating damage fronts is achieved in spite of the small amount of elements used in the discretization. Moreover the results are clearly comparable with the reference solutions and always obtained with a drastic reduction in the computer time. The quality of the solution at the inception of localization depends on the mesh configuration at this specific time-step. Even though the ALE solution is not very accurate at this precise time because the mesh is too coarse, the formulation corrects this error at subsequent time steps.

In the ALE formulation, it is clear that the initial number of degrees of freedom is the important parameter that must be adjusted in order to achieve an accurate description of strain localization. 
If it is too low, the interpolation will be too coarse despite element size optimization. An elegant solution would be to combine the ALE formulation with mesh adaptive techniques available in the literature when the number of degrees of freedom needs to be increased.

\section{APPENDIX: ERROR EQUIDISTRIBUTION OVER EACH ELEMENT}

The optimality criterion for equidistribution of an error indicator over the domain presented in equation (15) is obtained in this section. For simplicity a one-dimensional case is studied.

Given an error indicator $\mathscr{K}$ over the spatial domain $x \in\left[x_{\text {ins }}, x_{\text {sup }}\right]$ the position of the mesh points must be determined in order to equalize the error over each element. It is important to notice that the referential co-ordinates $\chi$ remain fixed in the ALE formulation, and that the finite element mesh is identically related to the fixed referential domain. That is, each nodal point always has the same co-ordinate in the referential domain.

The objective here is to define a mapping between $\chi$ and $x$ at every instant. Once this mapping, $x(\chi)$, is defined, the spatial co-ordinates of each nodal will be known. If a linear mapping is considered.

$$
\frac{\chi-\chi_{\text {inf }}}{\chi_{\text {sup }}-\chi_{\text {inf }}}=\frac{x(\chi)-x_{\text {inf }}}{x_{\text {sup }}-x_{\text {inf }}}
$$

the so-called one-dimensional isoparametric or transfinite mapping is obtained, see Reference 29. The mesh discretization over $\left[\chi_{\text {inf }}, \chi_{\text {sup }}\right]$ is homothetically reproduced over $\left[x_{\text {inf }}, x_{\text {sup }}\right]$.

On the other hand, if the error indicator $\mathscr{K}(x)$ must be equidistributed over the spatial domain another mapping must be defined:

$$
\frac{\chi-\chi_{\text {inf }}}{\chi_{\text {sup }}-\chi_{\text {inf }}}=\frac{\int_{x_{\text {int }}}^{x(x)} \mathscr{X}(\xi) \mathrm{d} \xi}{\int_{x_{\text {inf }}}^{x_{\text {iap }}} \mathscr{K}(\xi) \mathrm{d} \xi}
$$

where the linear behaviour imposed in the referential co-ordinates must be followed by the RHS quotient. For example, a referential point in the middle of the domain $\left(\left(\chi_{\text {mid }}-\chi_{\text {inf }}\right)\right)$ $\left.\left(\chi_{\text {sup }}-\chi_{\text {inf }}\right)=\frac{1}{2}\right)$ implies an associated $x\left(\chi_{\text {mid }}\right)$ such that $\mathscr{K}(x)$ is half distributed before and after $x\left(\chi_{\text {mid }}\right)$. Finally, differentiating equation (27) once with respect to $\chi$,

$$
\mathscr{K}(x) \frac{\partial x}{\partial \chi}=\int_{x_{\text {int }}}^{x_{\text {wwp }}} \mathscr{X}(\xi) \mathrm{d} \xi
$$

and then twice respect to $\chi$, the desired differential equation is obtained:

$$
\frac{\partial}{\partial \chi}\left[\mathscr{K}(x) \frac{\partial x}{\partial \chi}\right]=0
$$

Remark 1. The generalization to higher dimensions is automatic. Equations (26)-(29) are repeated for each co-ordinate. In fact, the referential co-ordinates $\chi$ and the spatial $\mathbf{x}$ ones are always related by one-to-one mappings and $\chi$ always defines a set of co-ordinate lines over the spatial domain.

Remark 2. For simplicity, the previous equation has been developed for each instant independently. However, transient mappings, $x(\chi, t)$, and error indicators, $\mathscr{K}(x, t)$, can be considered exactly in the same manner and equation (29) would also be obtained. 
Remark 3. A large number of automatic mesh generation schemes are based on the so-called 'partial differential equations methods' where the mapping between a predefined grid and the desired domain is defined implicitly by a set of partial differential equations. The elliptic techniques, and among them the Laplacian method, are probably the best known. Equation (29) could fall under this category.

Remark 4. An upper bound for the ratio of the maximum over the minimum length of elements throughout the mesh is determined from equation (28). The derivative $\partial x / \partial \chi$ is a local measure of the element size, and the RHS of (28) stays constant for a given error indicator. Thus, $\max (\mathscr{K})$ $\min (\partial x / \partial \chi)=\min (\mathscr{K}) \max (\partial x / \partial \chi)$. In the present study the maximum and minimum values of the error indicator are easily deduced from equations (14) and $(16)$, and the ratio $\max (\mathscr{K}) / \min (\mathscr{K})=$ $1+a / b$ bounds the maximum over the minimum element size.

\section{REFERENCES}

1. Z. P. Bażant and T. Belytschko, 'Wave propagation in a strain softening bar', J. Eng. Mech. ASCE, 111(3), 381-389 (1985).

2. T. Belytschko, Z. P. Bažant, Y. Hyun and T. P. Chang, 'Strain softening materials and finite element solutions', Comput. Struct., 23, 163-180 (1986).

3. R. de Borst, 'Simulation of strain localization: a reappraisal of the cosserat continuum', Eng. Comput, 8, 317-332 (1991).

4. G. Pijaudier-Cabot and Z. P. Bažant, 'Nonlocal damage theory', J. Eng. Mech. ASCE, 113, 1512-1533 (1987).

5. R, de Borst and H. B. Mühlhaus, 'Gradient dependent plasticity: formulation and algorithmic aspects', Int. j. numer. methods eng. 35, 521-540 (1992).

6. D. Lasry and T. Belytschko, 'Localization limiters in transient problems', Int. J. Solids Struct., 24, $581-597$ (1988).

7. L. J. Sluys and R. de Borst, 'Wave propagation and localization in rate-dependent cracked medium. Model formulation and one dimensional example', Int. J. Solids Struct., 29, 2945-2958 (1992).

8. A. Huerta, G, Pijaudier-Cabot and L. Bodé, 'ALE formulation in nonlocal strain softening solids' in D. R. J. Owen et al. (eds.), Proc. 3rd Int. Conf. on Computational Plasticity, COMPLAS III, Pineridge Press, Swansea, 1992, pp. $2249-2268$.

9. A. Huerta and G. Pijaudier-Cabot, 'Discretization influence on the regularization by two localization limiters', $J$. Eng. Mech. ASCE, 120, 1198-1218 (1994).

10. M. Ortiz and J. J. Quigley IV, 'Adaptive mesh refinement in strain localization problems', Comput. Methods Appl. Mech. Eng., 90, 781-804 (1991).

11. M. Pastor, J. Peraire and O. C. Zienkiewicz, 'Adaptive remeshing for shear band localization problems', Arch. Appl. Mech., 61, 30-39 (1991).

12. O. C. Zienkiewicz and G. C. Huang, 'A note on localization phenomena and adaptive finite element analysis in forming processes', Comm. Appl. Numer. Methods, 6, 71-76 (1990).

13. T. Belytschko and J. Fish, 'Spectral superposition on finite elements for shear banding problems', in R. Gruber et al. (eds.), Proc. 5th Int. Symp. on Numer. Methods in Eng. Springer, Berlin 1989, pp. 19-29.

14. A. Huerta and F. Casadei, 'New ALE applications in nonlinear fast-transient soild dynamics', Eng. Comput., 11, $317-345$ (1994).

15. A. Huerta and W. K. Liu, 'Viscous flow with large free surface motion', Comput. Methods Appl. Mech. Eng., 69, $277-324$ (1988)

16. W. K. Liu, T. Belytschko and H. Chang, 'An arbitrary Lagrangian-Eulerian finite element method for path dependent materials', Comput. Methods Appl. Mech. Eng., 58, 227-246 (1986).

17. W. K. Liu, H. Chang, J. S. Chen and T, Belytschko, 'Arbitrary Lagrangian-Eulerian stress update for forming simulations', Proc. Symp. on Recent Advances in Inelastic Analysis, ASME Winter Ann. Meeting, Boston, MA, 1987.

18. W. K. Liu, H. Chang, J. S. Chen and T. Belytschko, 'Arbitrary Lagrangian-Eulerian Petrov-Galerkin finite elements for nonlinear continua', Comput. Methods Appl. Mech. Eng., 68, 259-310 (1988).

19. T, Belytschko, J. M. Kennedy and D. F. Schoeberie, 'Quasi-Eulerian finite element formulation for fluid structure interaction', J. Pressure Vessel Technol. ASME, 102, 62-69 (1980).

20. J. Donéa, 'Arbitrary Lagrangian-Eulerian finite element methods', in T. Belytschko and T. J. R. Hughes (eds.), Computational Methods for Transient Analysis, Elsevier, Amsterdam 1983, pp. 473-516.

21. T. J. R. Hughes, W. K. Liu and T. K. Zimmerman, 'Lagrangian-Eulerian finite element formulation for incompressible viscous flows', Comput. Methods Appl. Mech. Eng., 29, 329-349 (1981).

22. J. Huétink, P. T. Vreede and J. Van Der Lugt, 'Progress in mixed Eulerian-Lagrangian finite element simulation of forming processes', Int. j. numer. methods eng., 30, 1441-1457 (1990). 


\section{ALE IN TRANSIENT PROBLEMS}

23. I. Babuška, and W, C. Rheinboldt, 'Error estimates for adaptive finite element computation', SIAM J. Numer. Anal., 15, 736-754 (1978).

24. P. Ladevẻze, J.-P. Pelle and $\mathrm{Ph}$. Rougeot, 'Error estimation and mesh optimization for classical finite elements', Eng. Comput., 8, 69-80 (1991).

25. O. C. Zienkiewicz and J. Z. Zhu, 'A simple error estimator and adaptive procedure for practical engineering analysis', Int. j. numer, methods eng., 24, 337-357 (1987).

26. J. Peraire, M. Vahdati, K. Morgan and O. C. Zienkiewicz, 'Adaptive remeshing for compressible flow computations', J. Comput. Phys., 72, 449-466 (1991).

27. E. Oñate and G. Bugeda, 'A study of mesh optimality criteria in adaptive finite element analysis', Eng. Comput., 10, 307-321 (1993).

28. J. Yu, D. Peric and D. R. J. Owen, Adaptive finite element analysis of a strain localisation problem for the elastoplastic cosserat continuum', in D. R. J. Owen et al. (eds.), Proc. 3rd Int. Conf. on Computational Plasticity, COMPLAS III, Pineridge Press, Swansea, 1992, pp. 551-566.

29. P. L. George, Automatic Mesh Generation: Application to Finite Element Methods, Wiley, New York, 1991. 